\title{
Accuracy of F-18 FDG PET/CT with optimal cut-offs of maximum standardized uptake value according to size for diagnosis of regional lymph node metastasis in patients with rectal cancer
}

Sung Uk Bae', Kyoung Sook Won², Bong-II Song², Woon Kyung Jeong ${ }^{1}$, Seong Kyu Baek ${ }^{1}$ and Hae Won Kim² ${ }^{2^{*}}$

\begin{abstract}
Background: The low sensitivity of F-18 fluorodeoxyglucose (FDG) positron emission tomography/computed tomography (PET/CT) for the evaluation of metastatic lymph nodes (LNS) is mainly due to the partial volume effect in patients with rectal cancer. This retrospective study evaluated the diagnostic accuracy of F-18 FDG PET/CT with optimal cut-off values of the maximum standardized uptake value ( $\left.S U V_{\text {max }}\right)$, according to $L N$ size, for the evaluation of regional LN in rectal cancer patients.
\end{abstract}

Methods: This study included 176 patients with rectal cancer who underwent F-18 FDG PET/CT for initial staging. Patients were classified based on the long-axis diameter of the regional $L N$ on $C T$ images as small $(\leq 7 \mathrm{~mm}$; $n=118)$ and large (> $7 \mathrm{~mm} ; n=58) \mathrm{LN}$ groups. The optimal cut-off value of SUV $V_{\max }$ was determined for each group, using receiver operating characteristic curve analysis. Areas under the curve (AUC) were compared by C-statistics using two methods: the cut-off value of SUV $\max$ optimized according to LN size, and a fixed SUV $V_{\max }$ Cut-off value of 2.5 .

Results: The optimal cut-off values of SUV $\max$ for the small and large LN groups were 1.1, and 2.1, respectively. The sensitivity, specificity, and accuracy of F-18 FDG PET/CT using the optimal cut-off values were 90.6, 70.9, and 76.3\% in the small LN group, and 68.6, 78.3, and $72.4 \%$ in the large LN group. The sensitivity, specificity, and accuracy of F-18 FDG PET/CT using the fixed cut-off value were 18.8, 100, and 78.0\% in the small LN group, and 51.4, 87.0, and $65.5 \%$ in the large LN group. The AUC was significantly higher using the optimal cut-off values than the fixed cut-off value $(0.808$ vs. $0.594, p=0.005)$ in the small LN group, but not in the large LN group (0.734 vs. 0.692, $p=0.429$ ).

Conclusions: Application of the lower cut-off value of SUV $\max$ improves the diagnostic performance of F-18 FDG PET/CT for the evaluation of small regional LNs in patients with rectal cancer.

Keywords: Rectal cancer, Lymph node metastasis, Maximum standardized uptake value, Partial volume effect, F-18 FDG, PET/CT

\footnotetext{
* Correspondence: hwkim.nm@gmail.com

${ }^{2}$ Department of Nuclear Medicine, Keimyung University Dongsan Medical

Center, 56 Dalseong-ro, Jung-gu, Daegu 41931, Republic of Korea

Full list of author information is available at the end of the article
}

(c) The Author(s). 2018 Open Access This article is distributed under the terms of the Creative Commons Attribution 4.0 International License (http://creativecommons.org/licenses/by/4.0/), which permits unrestricted use, distribution, and reproduction in any medium, provided you give appropriate credit to the original author(s) and the source, provide a link to the Creative Commons license, and indicate if changes were made. The Creative Commons Public Domain Dedication waiver (http://creativecommons.org/publicdomain/zero/1.0/) applies to the data made available in this article, unless otherwise stated. 


\section{Background}

Globally, colorectal cancer is the second most common cancer in women and the third most common cancer in men [1]. In Korea, the rectum was the most common site of cancer among both men and women in 1999 and again in 2009 [2]. Lymph node (LN) metastasis is one of the most important prognostic factors for patients with rectal cancer [3]. Additionally, LN metastasis plays a primary role in the determination of the operability and the extent of LN dissection. Survival is directly related to the presence of residual metastatic LNs after the primary operation. The accurate diagnosis of LN metastasis in initial staging may improve the prognosis and allow the early use of second-line therapy in patients with rectal cancer [4].

Conventional computed tomography (CT) and magnetic resonance imaging (MRI) have been commonly used for LN staging in patients with rectal cancer. However, both CT and MRI are limited by low sensitivity in the evaluation of small metastatic LNs [5-8]. Recently, F-18 fluorodeoxyglucose (FDG) positron emission tomography/computed tomography (PET/CT) has been proven to be useful for the preoperative staging of rectal cancer by revealing metabolic information of the lesion [9-11]. However, F-18 FDG PET/CT has also shown low sensitivity for the detection of LN metastasis [12, 13]. The low sensitivity of F-18 FDG PET/CT in the evaluation of metastatic LNs is mainly due to the partial volume effect, which spills out of the radioactivity into the background of small lesions $<10 \mathrm{~mm}$ in size, leading to underestimation of the true standardized uptake value (SUV) [14-16].

Several methods have been developed to correct the partial volume effect, and have significantly improved the diagnostic accuracy of metastatic LNs [17, 18]. However, there have been several limitations of the clinical use of partial volume correction due to the complexity of the method. Any method to consider size differences of LNs on F-18 FDG PET/CT images must be practical. Previous studies of an F-18 FDG PET/CT quantitative approach used a fixed cut-off of the maximum standardized uptake value $\left(\mathrm{SUV}_{\max }\right)$ in the diagnosis of LN metastasis, without considering the size differences of the LNs. Application of optimal $\mathrm{SUV}_{\text {max }}$ cut-off values according to LN size may improve the sensitivity of F-18 FDG PET/CT and may be practically useful for evaluation of the regional LNs in patients with rectal cancer. Thus, the aim of this study was to evaluate the diagnostic accuracy of F-18 FDG PET/CT using optimal SUV max $_{\text {cut-off values }}$ according to $\mathrm{LN}$ size to evaluate regional LNs in patients with rectal cancer.

\section{Subjects and methods}

\section{Study population}

We retrospectively analyzed the medical records of patients who underwent preoperative F-18 FDG PET/CT followed by curative operations for rectal cancer at our institution between January 2009 and August 2016. We excluded patients who underwent preoperative chemoradiation therapy and those with an interval of $>4$ weeks between F-18 FDG PET/CT and surgery. A retrospective cross-sectional analysis was performed to review the surgical and pathological findings and the F-18 FDG PET/CT results. Patients were classified based on the long-axis diameter of the regional $\mathrm{LN}$ on $\mathrm{CT}$ images as small $(\leq 7 \mathrm{~mm} ; n=118)$ and large $(>7 \mathrm{~mm} ; n=58) \mathrm{LN}$ groups. The reference value for long-axis diameter was determined as $7 \mathrm{~mm}$, because the partial volume effect is significant when the target of interest is smaller than 2 times of the PET/CT system's full-width at half-maximum (FWHM) $(<8 \mathrm{~mm})$ [18], and the long-axis diameter range on multiple detector $\mathrm{CT}$ has been reported as $7-10 \mathrm{~mm}$ for the diagnosis of metastatic regional LN [19, 20]. This study was approved by the Institutional Review Board of our institution.

\section{Histopathologic examination}

All surgeries were performed by qualified, experienced colorectal surgeons. Mesorectal excisions were performed in all patients; extended LN dissections were only performed if metastatic LNs were detected in frozen biopsies. All resected LNs underwent histopathologic exams for pathologic confirmation while labeling the exact location. The sensitivity, specificity, and accuracy of F-18 FDG PET/CT were calculated using the histopathologic result as the gold standard. A true positive was defined as a match between the location of the metastatic $\mathrm{LN}$ on pathologic examination and the location of the positive LN on an F-18 FDG PET/ CT image.

\section{F-18 FDG petPET/CT}

Two different F-18 FDG PET/CT systems were used (Discovery STE 16, GE Healthcare, Milwaukee, WI, USA; and Biograph mCT 64, Siemens Healthcare, Knoxville, TN, USA). The patients were required to fast for $>6 \mathrm{~h}$ before the scan, and the blood glucose level was measured to confirm that the level was $<180 \mathrm{mg} / \mathrm{dL}$ before injecting the F-18 FDG. In patients with diabetes, administration of antihyperglycemic drugs was stopped $12 \mathrm{~h}$ before the scan. Patients received intravenous administration of $4.0 \mathrm{MBq} / \mathrm{kg}$ (Biograph $\mathrm{mCT}$ ) and 7.0 MBq/kg (Discovery STE) F-18 FDG according to the $\mathrm{PET} / \mathrm{CT}$ system. Patients were encouraged to rest during the F-18 FDG uptake period. Images were acquired 
60 min after F-18 FDG administration. A non-contrast CT scan was obtained for attenuation correction and localization. Immediately after the CT scan, PET images were acquired from the base of the skull or top of the brain to the proximal thigh. The Discovery STE-16 PET/ CT scanner acquired images with a slice thickness of $3.75 \mathrm{~mm}$ simultaneously for a longitudinal field of view (FOV) of $780 \mathrm{~mm}$. The transaxial FOV was $70 \mathrm{~cm}$, and the matrix size was $128 \times 128$. Spatial resolution in air was $4.29 \mathrm{~mm}$ FWHM. The PET images were reconstructed from CT data for attenuation correction using the OSEM iterative algorithm with 20 subsets and two iterations. The Biograph mCT-64 PET/CT scanner acquired images with a slice thickness of $3 \mathrm{~mm}$ simultaneously for a longitudinal FOV of $500 \mathrm{~mm}$. The transaxial FOV was $58.8 \mathrm{~cm}$, and the matrix size was $256 \times 256$. Spatial resolution in air was $4 \mathrm{~mm}$ FWHM. The PET images were reconstructed from CT data for attenuation correction using the TrueX algorithm and an all-pass filter with 21 subsets and two iterations.

An experienced nuclear physician blinded to the histopathologic and colonoscopic results reviewed the F-18 FDG PET/CT images on a workstation (Advantage Workstation version 4.3; GE Healthcare). The locations of the regional LNs were recorded as the perirectal, superior rectal, inferior mesenteric, or internal iliac areas. Suspicious lymph nodes less than $3 \mathrm{~mm}$ were ignored because they cannot be differentiated from vascular structures or other nonspecific soft tissue densities. The ROIs (long-axis diameter range, $3-17 \mathrm{~mm}$ ) were drawn in consensus around the regional LNs, and the $\mathrm{SUV}_{\max }$ was measured using each dedicated PET workstation (ADW version 4.3 for Discovery STE-16 and syngo MI for Biograph mCT-64). The optimal $\mathrm{SUV}_{\max }$ cut-off values were determined using receiver operating characteristic curve (ROC) analysis for the small and large LN groups. When the measured $\mathrm{SUV}_{\max }$ exceeded the optimal cut-off value or 2.5, the LN was considered positive. In addition, subgroup analyses were performed according to the PET/CT scanner (PET A and B), T stage (early and advanced T stages), and F-18 FDG uptake of the primary tumor (low and high tumor $\mathrm{SUV}_{\max }$ ). Patients who were examined using the Discovery STE-16 PET/CT scanner were classified into the PET A group and patients who were examined using the Biograph mCT-64 $\mathrm{PET} / \mathrm{CT}$ scanner were classified into the PET B group. Patients with $\mathrm{T} 1$ or $\mathrm{T} 2$ stage were classified into the early $\mathrm{T}$ stage group and patients with $\mathrm{T} 3$ or $\mathrm{T} 4$ stage were classified into the advanced $\mathrm{T}$ stage group. Patients with $\mathrm{SUV}_{\max }$ of the primary tumor lower than 13.0, which was the median value of $\mathrm{SUV}_{\max }$, were classified into the low tumor $\mathrm{SUV}_{\max }$ group and patients with $\mathrm{SUV}_{\max }$ of the primary tumor higher than 13.0 were classified into the high tumor $\mathrm{SUV}_{\max }$ group. The optimal SUV $\mathrm{max}_{\max }$ cut-off values were determined for each subgroup.

\section{Statistical analyses}

The optimal cut-off values of the $\mathrm{SUV}_{\max }$ in each group and each subgroup were calculated using ROC analysis. The sensitivities, specificities, and accuracies of PET/CT using the optimal $\mathrm{SUV}_{\max }$ cut-off values according to LN size, and a fixed $\mathrm{SUV}_{\max }$ cut-off value of 2.5 , were calculated for each group, each subgroup, and for all patients together. The areas under the curve (AUCs) of the optimal and fixed $\mathrm{SUV}_{\max }$ cut-off values were compared using $\mathrm{C}$-statistics. A $p$-value $<0.05$ was considered significant.

\section{Results}

\section{Patient characteristics}

Of 296 patients who underwent preoperative F-18 FDG $\mathrm{PET} / \mathrm{CT}$ and follow-up curative surgery for rectal cancer, 120 patients were excluded from this study according to the exclusion criteria (Fig. 1). A total of 176 patients were included. Table 1 summarizes the patient characteristics. Patients were classified into the small $(n=118)$ or large $(n=58)$ LN groups. Regional LN metastasis was confirmed pathologically in 32 patients $(27.1 \%)$ in the small LN group, and 35 patients (60.3\%) in the large LN group.

The $\mathrm{SUV}_{\max }$ of large LNs was significantly higher than that of small LNs in the overall patient analysis (3.2 vs. $1.2, p<0.001)$. There was no significant difference in the SUV $_{\text {max }}$ of small and large LNs between the PET A and B groups (1.2 vs. $1.2, p=0.964$ and 2.6 vs. $3.7, p=210$ ). The $\mathrm{SUV}_{\max }$ of the small LN in the advanced $\mathrm{T}$ stage group was significantly higher than that in the early $\mathrm{T}$ stage group (1.5 vs. $1.0, p<0.001)$, but the $\mathrm{SUV}_{\max }$ of large LN was not significantly different between these two groups (3.6 vs. 2.0, $p=0.110$ ). The $\mathrm{SUV}_{\max }$ of the small LN in the high tumor $\mathrm{SUV}_{\max }$ group was significantly higher than that in the low tumor $\mathrm{SUV}_{\max }$ group (1.0 vs. $1.4, p<0.016$ ), but the $\mathrm{SUV}_{\max }$ of large LN was not significantly different between these two groups (3.6 vs. $2.5, p=0.230$ ).

\section{Accuracy of F-18 FDG PET/CT}

The optimal cut-off values of $\mathrm{SUV}_{\max }$ for the diagnosis of regional LN metastasis were 1.1 in the small LN group, and 2.1 in the large LN group. The sensitivity, specificity, accuracy, and AUC using the optimal SUV $\max$ cut-off values were $90.6,70.9,76.3 \%$, and 0.808 in the small LN group, and 68.6, 78.3, 72.4\%, and 0.734 in the large LN group, respectively (Table 2). Using the fixed $\mathrm{SUV}_{\max }$ cut-off value of 2.5 , the corresponding values were $18.8,100,78.0 \%$, and 0.594 in the small LN group, 


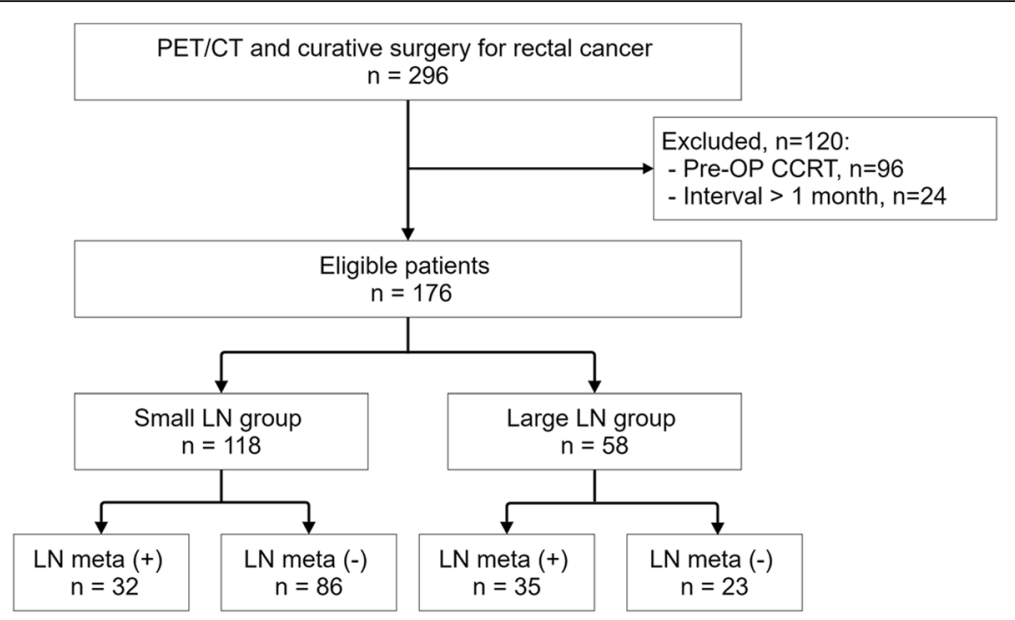

Fig. 1 Patient STARD flow chart

and 51.4, 87.0, 65.5\%, and 0.692 in the large LN group, respectively. The AUCs of PET/CT using the optimal cut-off values were significantly higher than those using the fixed cut-off value of 2.5 in the small $\mathrm{LN}$ group $(p=0.005)$. Figure 2 shows a representative

Table 1 Patient characteristics

\begin{tabular}{|c|c|c|c|}
\hline \multirow[t]{2}{*}{ Characteristics $^{\mathrm{a}}$} & \multirow{2}{*}{$\begin{array}{l}\text { Overall } \\
(n=176)\end{array}$} & \multicolumn{2}{|l|}{ Group $^{b}$} \\
\hline & & $\begin{array}{l}\text { Small LN } \\
(n=118)\end{array}$ & $\begin{array}{l}\text { Large LN } \\
(n=58)\end{array}$ \\
\hline Age, years & $66.7(10.4)$ & $67.4(9.3)$ & $65.3(12.4)$ \\
\hline Male, \% & 56.8 & 61 & 48.3 \\
\hline \multicolumn{4}{|l|}{ AJCC $^{\mathrm{d}}$ Stage, $n$} \\
\hline । & 69 & 59 & 10 \\
\hline$\|$ & 38 & 27 & 11 \\
\hline III & 69 & 32 & 37 \\
\hline IV & 0 & 0 & 0 \\
\hline LN diameter, mm & $6.2(3.0)$ & $4.6(1.7)$ & $9.4(2.5)$ \\
\hline$S U V_{\max }$ of $L N$ & $1.8(2.2)$ & $1.2(0.7)$ & $3.2(3.2)$ \\
\hline \multicolumn{4}{|l|}{ PET/CT scanner, $n$} \\
\hline Discovery STE-16 & 79 & 54 & 25 \\
\hline Biograph mCT-64 & 97 & 64 & 33 \\
\hline \multicolumn{4}{|l|}{ T stage, $\mathrm{n}$} \\
\hline $\mathrm{T} 1-2$ & 81 & 68 & 13 \\
\hline T3-4 & 95 & 50 & 45 \\
\hline \multicolumn{4}{|l|}{$\mathrm{J}_{\max }$ of primary tumor, $\mathrm{n}$} \\
\hline High SUV $\max (<13.0)$ & 84 & 64 & 20 \\
\hline LoW SUV $\max (>13.0)$ & 92 & 54 & 38 \\
\hline
\end{tabular}

${ }^{a}$ All values are presented as means (SD)

${ }^{\mathrm{b}}$ Patients were categorized by the long-axis diameter of the regional LN, as follows: small $\mathrm{LN}, \leq 7 \mathrm{~mm}$; large $\mathrm{LN},>7 \mathrm{~mm}$ 'LN lymph node

${ }^{\mathrm{d}}$ AJCC American Joint Committee on Cancer case of regional LN metastasis that was predicted by using the optimal $\mathrm{SUV}_{\max }$ cut-off values, but not by the fixed $\mathrm{SUV}_{\max }$ cut-off value of 2.5. There was no significant difference in the AUC between the two methods in the large LN group $(p=0.429)$.

In overall patients, the sensitivity, specificity, accuracy, and AUC of F-18 FDG PET/CT, using the optimal cut-off values, were $76.1,74.3,75.0 \%$, and 0.752 , respectively whereas on using the fixed cut-off value, the sensitivity, specificity, accuracy, and AUC were 35.8, 97.2, $73.9 \%$, and 0.665 , respectively. The AUC of PET/CT using the optimal cut-off value was higher than that using the fixed cut-off value of 2.5 in all patients, but not statistically significant $(p=0.071)$.

Subgroup analysis was performed according to the PET/CT scanner. In the PET A group, the optimal cut-off values of $\mathrm{SUV}_{\max }$ were 1.1 for small $\mathrm{LN}$ and 2.1 for large LN. In the PET B group, the optimal cut-off values of $\mathrm{SUV}_{\max }$ were 1.0 for small $\mathrm{LN}$ and 1.9 for large LN. Table 3 shows the sensitivity, specificity, accuracy, and AUC in the PET A and B groups. In the small LN of the PET A group, the AUC using the optimal cut-off value was significantly higher than that using the fixed cut-off value of $2.5(p=0.047)$. There were no significant differences in the AUCs between PET/CT using the optimal and fixed cut-off values in the large LN of the PET A group ( $p=0.866)$, as well as small and large LNs of the PET B group ( $p=0.110$ and $p=0.162)$. Subgroup analysis according to the $\mathrm{T}$ stage revealed that the optimal cut-off values were 0.9 for small $\mathrm{LN}$ and 1.8 for large LN in the early T stage group and 1.1 for small LN and 2.1 for large $\mathrm{LN}$ in the advanced $\mathrm{T}$ stage group. There were no significant differences in the AUCs between PET/CT using the optimal and fixed cut-off values in the small and large LNs of the early and advanced $\mathrm{T}$ stage groups $(p=0.188, p=1.000, p=0.231$ 
Table 2 Comparison of the diagnostic values between PET/CT using the cut-off values of SUV $V_{\max }$ optimized according to the lymph node (LN) size and the fixed SUV $\max$ cut-off value of 2.5

\begin{tabular}{|c|c|c|c|c|c|c|c|c|}
\hline Group & Cut-off values & Sensitivity (\%) & Specificity (\%) & PPV $^{a}(\%)$ & NPV' (\%) & Accuracy (\%) & $A \cup C^{C}$ & $p$ \\
\hline \multirow[t]{2}{*}{ Overall } & 2.5 & 35.8 & 97.2 & 88.9 & 71.1 & 73.9 & 0.665 & \multirow[t]{2}{*}{0.071} \\
\hline & $\mathrm{Opt}^{\mathrm{d}}$ & 76.1 & 74.3 & 64.6 & 83.5 & 75 & 0.752 & \\
\hline \multirow[t]{2}{*}{ Small LN } & 2.5 & 18.8 & 100 & 100 & 76.8 & 78 & 0.594 & \multirow[t]{2}{*}{0.005} \\
\hline & 1.1 & 90.6 & 70.9 & 53.7 & 95.3 & 76.3 & 0.808 & \\
\hline \multirow[t]{2}{*}{ Large LN } & 2.5 & 51.4 & 87 & 85.7 & 54.1 & 65.5 & 0.692 & \multirow[t]{2}{*}{0.429} \\
\hline & 2.1 & 68.6 & 78.3 & 82.8 & 62.1 & 72.4 & 0.734 & \\
\hline
\end{tabular}

${ }^{a} P P V$ positive predictive value

${ }^{\mathrm{b}} \mathrm{NPV}$ negative predictive value

${ }^{c} A \cup C$ area under the curve

${ }^{\mathrm{d}}$ Opt optimal cut-off values of SUV $\max (1.1$ in the small LN group and 2.1 in the large LN group)

and $p=0.822$ ). Additional file 1 : Table $\mathrm{S} 1$ shows the sensitivity, specificity, accuracy, and AUC in the early and advanced T stage groups. Subgroup analysis according to the $\mathrm{SUV}_{\max }$ of the primary tumor revealed that the optimal cut-off values were 1.0 for small $\mathrm{LN}$ and 1.3 for large LN in the low tumor $\mathrm{SUV}_{\max }$ group, and 1.1 for small LN and 2.1 for large LN in the high tumor $\mathrm{SUV}_{\max }$ group. There were no significant differences in the AUCs between PET/CT using the optimal and fixed cut-off values in the small and large LNs of the low and high tumor $\mathrm{SUV}_{\max }$ groups $(p=0.070, p=0.908, p=$ 0.177 and $p=0.491$ ). Additional file 2: Table S2 shows the sensitivity, specificity, accuracy, and AUC in the low and high tumor SUV $\mathrm{max}_{\max }$ groups.

\section{Discussion}

The present study revealed improved diagnostic performance of F-18 FDG PET/CT in the evaluation of metastatic LNs in patients with rectal cancer using the optimal $\mathrm{SUV}_{\max }$ cut-off values according to the size of the LN. Application of a lower SUV $V_{\max }$ cut-off value to evaluate a small LN increased the sensitivity of PET/CT in the detection of metastatic LNs in patients with rectal cancer. The AUCs of the PET/CT with optimal SUV $\max$ cut-off values were significantly higher than those with a fixed cut-off value of 2.5 in the small LN group. These results suggest that F-18 FDG PET/CT can diagnose LN metastasis as accurately in small LNs as in large LNs if a lower $\mathrm{SUV}_{\max }$ cut-off value is applied. Although the concept of this hypothesis is widely known, the present study proved it practically in sufficient number of patients with rectal cancer.

LN metastasis in rectal cancer is directly correlated with prognosis. The 5 -year survival rate is $>95 \%$ in rectal cancer patients without LN metastasis, but decreases to $50 \sim 70 \%$ in patients with LN metastasis [3]. Additionally, the LN stage of rectal cancer is one of the most important determining factors for adjuvant chemotherapy and extended LN dissection [21, 22]. The procedure of choice for rectal cancer patients with a clinical stage of N0 or N1 is total mesorectal excision, which is surgical excision of the mesorectal fat, including all LNs. In more

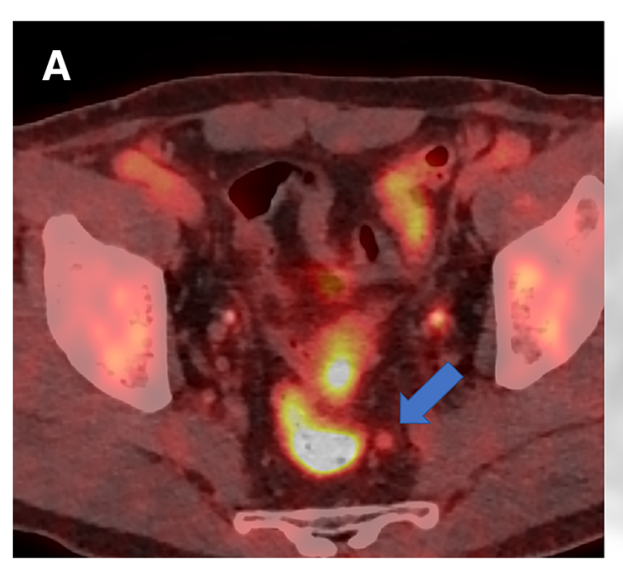

B

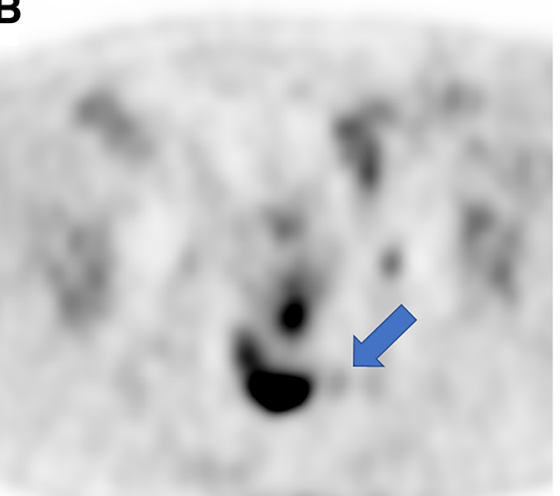

Fig. 2 A representative case of regional LN metastasis predicted by optimal SUV $\max$ Cut-off values, but not by the fixed SUV $V_{\max }$ Cut-off value of 2.5. (a) A mildly hypermetabolic lymph node (arrow) was observed in the left perirectal region. The long-axis diameter of the LN was $6 \mathrm{~mm}$, and the patient was classified into the small LN group according to the size criteria. (b) The SUV max of the LN was 1.8, and exceeded the optimal SUV $\max$ Cut-off value of 1.1. Histopathologic examination revealed that the lesion was a metastatic LN 
Table 3 Comparison of diagnostic values between PET/CT using the optimized cut-off values and the fixed cut-off value of 2.5 in patients imaged by PET $A$ and $B$

\begin{tabular}{|c|c|c|c|c|c|c|c|c|}
\hline Groups & Cut-off values & Sensitivity (\%) & Specificity (\%) & $\mathrm{PPV}^{\mathrm{a}}(\%)$ & NPV' (\%) & Accuracy (\%) & $A \cup C^{C}$ & $p$ \\
\hline \multicolumn{9}{|l|}{ PET A } \\
\hline \multirow[t]{2}{*}{ Overall } & 2.5 & 36.7 & 98.0 & 91.7 & 71.6 & 74.7 & 0.673 & 0.169 \\
\hline & Opt & 76.7 & 77.6 & 67.6 & 84.4 & 77.2 & 0.771 & \\
\hline \multirow[t]{2}{*}{ Small LN } & 2.5 & 18.8 & 100.0 & 100.0 & 74.5 & 75.9 & 0.594 & 0.047 \\
\hline & 1.1 & 87.5 & 73.7 & 58.3 & 93.3 & 77.8 & 0.806 & \\
\hline \multirow[t]{2}{*}{ Large LN } & 2.5 & 57.1 & 90.9 & 88.9 & 62.5 & 72.0 & 0.740 & 0.866 \\
\hline & 2.1 & 78.6 & 72.7 & 78.6 & 72.7 & 76.0 & 0.756 & \\
\hline \multicolumn{9}{|l|}{ PET B } \\
\hline \multirow[t]{2}{*}{ Overall } & 2.5 & 35.1 & 96.7 & 86.7 & 70.7 & 73.2 & 0.659 & 0.520 \\
\hline & Opt & 75.7 & 65.0 & 57.1 & 81.3 & 69.1 & 0.703 & \\
\hline \multirow[t]{2}{*}{ Small LN } & 2.5 & 18.8 & 100.0 & 100.0 & 78.7 & 79.7 & 0.594 & 0.110 \\
\hline & 1.0 & 93.8 & 60.4 & 44.1 & 96.7 & 68.8 & 0.771 & \\
\hline \multirow[t]{2}{*}{ Large LN } & 2.5 & 47.6 & 83.3 & 83.3 & 47.6 & 60.6 & 0.655 & 0.162 \\
\hline & 1.9 & 61.9 & 83.3 & 86.7 & 55.6 & 69.7 & 0.726 & \\
\hline
\end{tabular}

${ }^{a} P P V$ positive predictive value

${ }^{\mathrm{b}} \mathrm{NPV}$ negative predictive value

${ }^{\mathrm{C}} A \cup C$ area under the curve

${ }^{\mathrm{d}}$ Opt optimal cut-off values of SUV $\max$

advanced cancers with a clinical stage of N2, preoperative concurrent chemoradiotherapy is recommended. Extended LN dissection is required in patients with suspected metastatic LNs in the lateral pelvic region [23-25]. Application of the optimal $\mathrm{SUV}_{\max }$ cut-off values according to the LN size allows determination of treatment strategies and improves the prognosis of patients with rectal cancer by improving the accuracy of the diagnosis of LN metastasis with F-18 FDG $\mathrm{PET} / \mathrm{CT}$.

F-18 FDG PET/CT is beneficial in the preoperative staging of rectal cancer, though it showed low sensitivity and accuracy in the diagnosis of $\mathrm{LN}$ metastasis [12, 13]. There is no definite evidence supporting F-18 FDG $\mathrm{PET} / \mathrm{CT}$ as the routine clinical application in the evaluation of LN metastasis, though F-18 FDG PET/CT could be used to supplement the possibility of suspected metastatic LNs detected by other imaging modalities. For a quantitative approach to the diagnosis of LN metastasis on F-18 FDG PET/CT images, a fixed cut-off value of $\mathrm{SUV}_{\max }$ of 2.5 has been commonly used to diagnose metastatic LNs $[5,12]$. However, the sensitivity (38 65\%) of F-18 FDG PET/CT in the diagnosis of LN metastasis were low compared to those of CT and MRI [13]. In accordance with previous studies, the corresponding values using a fixed $\mathrm{SUV}_{\max }$ cut-off value of 2.5 in the present study were comparably low. However, there was significant improvement in the sensitivity of PET/CT when using an SUV max $_{\text {ax }}$ cut-off value optimized according to LN size.
The primary cause of the low sensitivity of F-18 FDG $\mathrm{PET} / \mathrm{CT}$ in the diagnosis of LN metastasis is the partial volume effect [14-16], which causes the underestimation of radioactivity concentration in structures with less than two to three times the spatial resolution of PET $(4 \sim 5 \mathrm{~mm})$. Due to the partial volume effect, the quantitative evaluation of LN metastasis has not been a routine practice in the interpretation of F-18 FDG PET/CT images. Due to the partial volume effect, lower optimal $\mathrm{SUV}_{\max }$ cut-off values were determined for the evaluation of small LNs than for the large LNs. Several techniques have been developed to calibrate the partial volume effect. In addition, many studies revealed significant improvement in the diagnostic performance of F-18 FDG PET/CT for the determination of small lesions after partial volume correction $[17,18]$. However, partial volume correction methods are generally too complex to be clinically applicable, and most require additional equipment or applications. Therefore, we applied the optimal SUV max $_{\text {max }}$ cut-off values according to LN size to compensate for the partial volume effect, which resulted in significant improvement in the AUC. This approach could be a more rapid and simpler method for calibration of the partial volume effect.

CT and MRI have conventionally been used to evaluate $\mathrm{LN}$ metastasis in rectal cancer by evaluating the size and shape of the $\operatorname{LN}[5,6]$. A diameter of $5 \sim 10 \mathrm{~mm}$ has been applied as the threshold to diagnose metastatic LNs, but many studies have revealed the limitations of using size criteria alone for LN staging in rectal cancer. 
Approximately $60 \%$ of metastatic LNs are $<5 \mathrm{~mm}$ in diameter [26]. Therefore, evaluating the shape of the LN can also be useful in diagnosis. In most metastatic LNs, the loss of the fatty hilum and kidney bean-shaped structure can be detected. A recent meta-analysis study including $12 \mathrm{CT}$ studies reported that the pooled sensitivity and specificity of CT for LN metastasis were 71\% and $67 \%$, respectively [27]. Another meta-analysis study including 21 MRI studies reported that the pooled sensitivity and specificity of MRI for LN metastasis were 77 and $71 \%$, respectively [28]. In the present study, F-18 FDG PET/CT using a fixed SUV $\max$ cut-off value of 2.5 showed low sensitivity (35.8\%) and high specificity (97.2\%), whereas F-18 FDG PET/CT using SUV $\max$ cut-off values optimized according to size showed high sensitivity $(76.1 \%)$ and high specificity $(74.3 \%)$. The diagnostic value of the present study is considered to be comparable to those of previous CT or MRI studies. However, direct comparison of diagnostic value is limited between the present study and previous CT or MRI studies, because there are heterogeneities between studies including differences in protocols, radiologists' experience, approach to image interpretation, and methodologic quality. Further studies with comparison of diagnostic value between F-18 FDG PET/CT, CT, and MRI in the same patient population could provide important information in selecting diagnostic modalities for preoperative staging of rectal cancer.

The limitation of the present study was the use of two different scanners (Discovery STE-16, GE Healthcare; and Biograph mCT-64, Siemens Healthcare), which could not be avoided owing to the retrospective study design. The difference in the resolution and administered dose of F-18 FDG, according to the two different scanners, could have caused differences in the $\mathrm{SUV}_{\max }$ and could have affected some of the results of the present study. However, previous studies have shown that the difference in the $S U V_{\text {max }}$ of the same lesion between two different scanners is $<0.05$ [29]. In the present study, there were no significant differences in the $\mathrm{SUV}_{\max }$ of small and large LNs between the two scanners. Furthermore, the difference between the optimal cut-off values of the two scanners was only 0.1 for small LNs. Although the effect of using two different scanners on the results of the present study would be negligible, further prospective studies involving the use of one PET/CT scanner and a large population are needed for more valid optimal cut-off values.

\section{Conclusions}

Application of the lower cut-off value of $\mathrm{SUV}_{\max }$ increases the sensitivity of F-18 FDG PET/CT for evaluation of the small regional LNs in patients with rectal cancer. F-18 FDG PET/CT using the optimized SUV $\max$ cut-off values according to the $\mathrm{LN}$ size has the potential to show improved diagnostic performance for the detection of regional LN metastasis in patients with rectal cancer. Further prospective studies involving the use of one PET/CT scanner and a large population are needed.

\section{Additional files}

\begin{abstract}
Additional file 1: Table S1. Comparison of diagnostic values between PET/CT using the optimized cut-off values and the fixed cut-off value of 2.5 in patients with early (T1-2) and advanced (T3-4) T stages. (DOCX 19 kb)

Additional file 2: Table S2. Comparison of diagnostic values between PET/CT using the optimized cut-off values and the fixed cut-off value of 2.5 in patients with low $(<13.0)$ and high $(>13.0)$ SUV $_{\max }$ of primary tumor. (DOCX 17 kb)
\end{abstract}

\section{Abbreviations \\ AUC: areas under the curve; CT: conventional computed tomography; FDG: fluorodeoxyglucose; LN: lymph node; MRI: magnetic resonance imaging; PET/CT: positron emission tomography/computed tomography; ROC: receiver operating characteristic curve; SUV: standardized uptake value; SUV $V_{\text {max }}$ : maximum standardized uptake value}

\section{Ethical approval and consent to participate}

All procedures performed in studies involving human participants were in accordance with the ethical standards of the institutional and/or national research committee and with the principles of the 1964 Declaration of Helsinki and its later amendments or comparable ethical standards. The institutional review board of Dongsan Medical Center approved this study (No. 2018-03-023), and written informed consent for study participation was obtained from all participants.

\section{Funding}

This study was supported by a National Research Foundation of Korea (NRF) grant funded by the Korea Government (MSIP) (no. 2014R1A5A2010008 and no. 2017R1C1B5017721)

\section{Availability of data and materials}

The dataset supporting the conclusions of this article is included within the article. Data and materials during the current study are available from the corresponding author upon reasonable request.

\section{Authors' contributions}

HWK and SUB participated in the design of the study, and drafted the manuscript. SUB, WKJ and SKB collected the patients'data. BWK and BS processed the figures, helped draft the manuscript, and performed a critical revision of the manuscript. KSW and SUB conceived and designed the study and supervised the project. All authors read and approved the final version of the manuscript.

\section{Consent for publication}

Written informed consent for publication was obtained from each patient.

\section{Competing interests}

The authors declare that they have no competing interests

\section{Publisher's Note}

Springer Nature remains neutral with regard to jurisdictional claims in published maps and institutional affiliations.

\section{Author details}

'Department of Surgery, Keimyung University Dongsan Medical Center, Daegu, Republic of Korea. ${ }^{2}$ Department of Nuclear Medicine, Keimyung University Dongsan Medical Center, 56 Dalseong-ro, Jung-gu, Daegu 41931 Republic of Korea. 
Received: 2 July 2018 Accepted: 30 August 2018

Published online: 14 September 2018

\section{References}

1. Shaukat A, Church TR. Colorectal-cancer incidence and mortality after screening. N Engl J Med. 2013;369(24):2355.

2. Shin A, Kim KZ, Jung KW, Park S, Won YJ, Kim J, et al. Increasing trend of colorectal cancer incidence in Korea, 1999-2009. Cancer Res Treat. 2012;44(4):219-26.

3. Zhou L, Wang JZ, Wang JT, WU YJ, Chen H, Wang WB, et al. Correlation analysis of MR/CT on colorectal cancer lymph node metastasis characteristics and prognosis. Eur Rev Med Pharmacol Sci. 2017:21(6):1219-25.

4. Glynne-Jones R, Wyrwicz L, Tiret E, Brown G, Rodel C, Cervantes A, et al. Rectal cancer: ESMO Clinical Practice Guidelines for diagnosis, treatment and follow-up. Ann Oncol. 2017;28(suppl_4):iv22-40.

5. Tateishi U, Maeda T, Morimoto T, Miyake M, Arai Y, Kim EE. Non-enhanced $C T$ versus contrast-enhanced $C T$ in integrated PET/CT studies for nodal staging of rectal cancer. Eur J Nucl Med Mol Imaging. 2007;34(10):1627-34.

6. Bipat S, Glas AS, Slors FJ, Zwinderman AH, Bossuyt PM, Stoker J. Rectal cancer: local staging and assessment of lymph node involvement with endoluminal US, CT, and MR imaging--a meta-analysis. Radiology. 2004;232(3):773-83.

7. Kim NK, Kim MJ, Yun SH, Sohn SK, Min JS. Comparative study of transrectal ultrasonography, pelvic computerized tomography, and magnetic resonance imaging in preoperative staging of rectal cancer. Dis Colon Rectum. 1999:42(6):770-5.

8. Park IJ, Kim HC, Yu CS, Ryu MH, Chang HM, Kim JH, et al. Efficacy of PET/CT in the accurate evaluation of primary colorectal carcinoma. Eur J Surg Oncol. 2006;32(9):941-7.

9. Kijima S, Sasaki T, Nagata K, Utano K, Lefor AT, Sugimoto H. Preoperative evaluation of colorectal cancer using CT colonography, MRI, and PET/CT. World J Gastroenterol. 2014;20(45):16964-75.

10. Abdel-Nabi H, Doerr RJ, Lamonica DM, Cronin VR, Galantowicz PJ, Carbone $\mathrm{GM}$, et al. Staging of primary colorectal carcinomas with fluorine-18 fluorodeoxyglucose whole-body PET: correlation with histopathologic and CT findings. Radiology. 1998;206(3):755-60.

11. Kantorova I, Lipska L, Belohlavek O, Visokai V, Trubac M, Schneiderova M. Routine (18)F-FDG PET preoperative staging of colorectal cancer: comparison with conventional staging and its impact on treatment decision making. J Nucl Med. 2003:44(11):1784-8.

12. Tsunoda Y, Ito M, Fujji H, Kuwano H, Saito N. Preoperative diagnosis of lymph node metastases of colorectal cancer by FDG-PET/CT. Jpn J Clin Oncol. 2008;38(5):347-53.

13. Brush J, Boyd K, Chappell F, Crawford F, Dozier M, Fenwick E, et al. The value of FDG positron emission tomography/computerised tomography (PET/CT) in pre-operative staging of colorectal cancer: a systematic review and economic evaluation. Health Technol Assess. 2011;15(35):1-192. iii-iv

14. Rahmim A, Qi J, Sossi V. Resolution modeling in PET imaging: theory, practice, benefits, and pitfalls. Med Phys. 2013;40(6):064301.

15. Soret M, Bacharach SL, Buvat I. Partial-volume effect in PET tumor imaging. J Nucl Med. 2007:48(6):932-45.

16. Steinert HC, Hauser M, Allemann F, Engel $H$, Berthold $T$, von Schulthess GK, et al. Non-small cell lung cancer: nodal staging with FDG PET versus CT with correlative lymph node mapping and sampling. Radiology. 1997;202(2):441-6.

17. Boussion N, Cheze Le rest C, Hatt M, Visvikis D. Incorporation of waveletbased denoising in iterative deconvolution for partial volume correction in whole-body PET imaging. Eur J Nucl Med Mol Imaging. 2009;36(7):1064-75.

18. Bettinardi V, Castiglioni I, De Bernardi E, Gilardi M. PET quantification: strategies for partial volume correction. Clin Transl Imaging. 2014;2(3):199-218.

19. Inoue $Y$, Saigusa $S$, Hiro J, Toiyama $Y$, Araki T, Tanaka $K$, et al. Clinical significance of enlarged lateral pelvic lymph nodes before and after preoperative chemoradiotherapy for rectal cancer. Mol Clin Oncol. 2016;4(6):994-1002.

20. Rollven E, Abraham-Nordling M, Holm T, Blomqvist L. Assessment and diagnostic accuracy of lymph node status to predict stage III colon cancer using computed tomography. Cancer Imaging. 2017;17(1):3.
21. Choi PW, Kim HC, Kim AY, Jung SH, Yu CS, Kim JC. Extensive lymphadenectomy in colorectal cancer with isolated Para-aortic lymph node metastasis below the level of renal vessels. J Surg Oncol. 2010;101(1):66-71.

22. Song SH, Park SY, Park JS, Kim HJ, Yang CS, Choi GS. Laparoscopic Paraaortic lymph node dissection for patients with primary colorectal cancer and clinically suspected Para-aortic lymph nodes. Ann Surg Treat Res. 2016;90(1):29-35.

23. Akasu T, Sugihara K, Moriya Y. Male urinary and sexual functions after mesorectal excision alone or in combination with extended lateral pelvic lymph node dissection for rectal cancer. Ann Surg Oncol. 2009;16(10):2779-86

24. Kim DJ, Chung JJ, Yu JS, Cho ES, Kim JH. Evaluation of lateral pelvic nodes in patients with advanced rectal cancer. AJR Am J Roentgenol. 2014:202(6):1245-55

25. Yano H, Saito Y, Takeshita E, Miyake O, Ishizuka N. Prediction of lateral pelvic node involvement in low rectal cancer by conventional computed tomography. Br J Surg. 2007;94(8):1014-9.

26. Brown G, Richards CJ, Bourne MW, Newcombe RG, Radcliffe AG, Dallimore NS, et al. Morphologic predictors of lymph node status in rectal cancer with use of high-spatial-resolution MR imaging with histopathologic comparison. Radiology. 2003;227(2):371-7.

27. Nerad E, Lahaye MJ, Maas M, Nelemans P, Bakers FC, Beets GL, et al. Diagnostic accuracy of $\mathrm{CT}$ for local staging of Colon Cancer: a systematic review and meta-analysis. Am J Roentgenol. 2016;207(5):984-95.

28. Al-Sukhni E, Milot L, Fruitman M, Beyene J, Victor JC, Schmocker S, et al. Diagnostic accuracy of MRI for assessment of T category, lymph node metastases, and circumferential resection margin involvement in patients with rectal cancer: a systematic review and meta-analysis. Ann Surg Oncol. 2012;19(7):2212-23.

29. Sunderland JJ, Christian PE. Quantitative PET/CT scanner performance characterization based upon the society of nuclear medicine and molecular imaging clinical trials network oncology clinical simulator phantom. J Nucl Med. 2015;56(1):145-52.

Ready to submit your research? Choose BMC and benefit from:

- fast, convenient online submission

- thorough peer review by experienced researchers in your field

- rapid publication on acceptance

- support for research data, including large and complex data types

- gold Open Access which fosters wider collaboration and increased citations

- maximum visibility for your research: over $100 \mathrm{M}$ website views per year

At BMC, research is always in progress.

Learn more biomedcentral.com/submissions 